\title{
Historia de las infecciones por coronavirus y epidemiología de la infección por SARS-CoV-2
}

\author{
History of coronavirus infections and epidemiology \\ of SARS-CoV-2 infection
}

\author{
Ricardo Iván Velázquez-Silva* \\ * Área de Nefrología del Trasplante. Departamento de Nefrología y Metabolismo Mineral. \\ Instituto Nacional de Ciencias Médicas y Nutrición «Salvador Zubirán». Ciudad de México, México.
}

\section{RESUMEN}

La primera descripción del coronavirus (CoV) humano fue en 1965 por Tyrrell y se llamó así debido a las proyecciones desde su superficie que semejaban a una corona. Los CoV suelen causar enfermedades respiratorias y gastrointestinales en humanos. Las primeras epidemias fueron causadas por el SARS-CoV (Severe Acute Respiratory Syndrome-CoV) en el 2002 y por el MERS-CoV (Middle East Respiratory Syndrome-CoV) en 2012. La epidemia actual es causada por el nuevo coronavirus llamado SARS-CoV-2 causante de la enfermedad COVID-19. Los primeros casos se reportaron en Wuhan, China, en diciembre del 2019 y desde entonces la propagación de la enfermedad ha sido global. La Organización Mundial de la Salud (OMS) la declaró pandemia el 11 de marzo del 2020. Para el 27 de mayo se han reportado 5,488,825 casos confirmados de SARS-CoV-2 en todo el mundo, el continente más afectado es el americano con $2,495,924$ casos, seguido de Europa con 2,061,828 casos. En México, el primer caso fue diagnosticado el 28 de febrero de 2020. Inicialmente en China se reportó que el $14 \%$ de los pacientes se presentaban con sintomatología moderada, $4.7 \%$ pacientes en estado crítico y mortalidad del $2.3 \%$ con variabilidad en diferentes países. En México la mortalidad es más alta (9.2\%). La presentación de la enfermedad en pacientes con enfermedad renal crónica es atípica (diarrea en un tercio de los pacientes) y con mayor mortalidad (16.2-52\%). En pacientes receptores de trasplante renal la enfermedad se presenta con mayores complicaciones y mayor mortalidad (10-66\%).

Palabras clave: SARS-CoV-2, COVID-19, epidemiología, enfermedad renal crónica, hemodiálisis, receptor de trasplante renal.

\begin{abstract}
The first description of the human coronavirus (CoV) was in 1965 by Tyrrell and was so named because of crown-like projections from its surface. CoV's usually cause respiratory and gastrointestinal diseases in humans. The first epidemics were caused by SARS-CoV (Severe Acute Respiratory Syndrome CoV) in 2002 and by MERS-CoV (Middle East Respiratory Syndrome CoV) in 2012. The current epidemic is caused by the new coronavirus called SARS-CoV-2 causing of COVID-19 disease. The first cases were reported in Wuhan, China, in December 2019 and since then the spread of the disease has been global. The World Health Organization declared it a pandemic on March 11, 2020. By May 27, a total of 5,488,825 confirmed cases of SARS-CoV-2 have been reported worldwide, the most affected continent is the American with 2,495,924 cases, followed by Europe with 2,061,828 cases. In Mexico, the first case was diagnosed on February 28, 2020. Initially in China it was reported that $14 \%$ of patients presented with moderate symptoms, $4.7 \%$ critically ill patients, and $2.3 \%$ mortality with variability in different countries. In Mexico mortality is higher (9.2\%). The presentation of the disease in patients with chronic kidney disease is atypical (diarrhea in one third of patients) and with higher mortality (16.2-52\%). In kidney transplant recipients, COVID-19 disease presents with greater complications and higher mortality (10-66\%).
\end{abstract}

Keywords: SARS-CoV-2, COVID-19, epidemiology, chronic kidney disease, hemodialysis, kidney transplant recipient. 
Historia de las infecciones

causadas por coronavirus

En las pasadas dos décadas, dos tipos de coronavirus (CoV) previamente desconocidos, el SARS-CoV (Severe Acute Respiratory Syndrome-CoV) y el MERSCoV (Middle East Respiratory Syndrome-CoV) acapararon la atención médica, científica y mediática debido a su naturaleza letal y alto potencial epidémico. Ahora la aparición de un tercer nuevo virus, el SARS-CoV-2, tiene al mundo en una situación de emergencia sanitaria, económica y social no vista desde la segunda guerra mundial.

La primera descripción de un coronavirus humano fue en 1965 por Tyrrell y Bynoe quienes nombraron al virus como B814. La presencia del agente infeccioso se demostró al inocular a voluntarios sanos con el medio de cultivo del virus y con la consecuente producción de enfermedad de vía respiratoria superior. ${ }^{1,2}$ Posteriormente Almeida y Tyrrell por medio de observaciones con microscopia electrónica del B814, describieron estructuras de tamaño medio $(80-150 \mathrm{~nm})$ con proyecciones desde su superficie que asemejaban una corona ${ }^{3}$ y en 1975 se bautizó a este virus como coronavirus. ${ }^{4}$

Antes del 2002, los coronavirus patógenos para el ser humano eran el CoV 229E (HCoV-229E) y HCoVOC4. Estos coronavirus se manifiestan clínicamente como infecciones respiratorias altas, leves, en pacientes adultos inmunocompetentes ${ }^{5}$ y como una infección más severa en niños, adultos mayores y pacientes inmunosuprimidos. ${ }^{6}$

\section{SARS-CoV}

Los CoV no se habían considerado como patógenos altamente virulentos hasta el brote de «neumonías atípicas» en noviembre del 2002 en la ciudad de Foshan de la provincia de Guangdong, China. ${ }^{7-11}$ Del 24 de enero al 18 de febrero de 2003 se hospitalizaron 55 pacientes con diagnóstico de «neumonía atípica», se encontró como patógeno causal al SARS-CoV en tres pacientes por medio de aislamiento en cultivo de hisopado nasal y serología positiva en 48 pacientes $(87 \%)^{7}$

El 11 de febrero de 2003, la OMS recibió el informe del ministerio de salud de China que reportó un brote de 300 casos de SARS y cinco muertes asociadas en la provincia de Guangdong, y para el 21 de febrero de 2003 la infección ya se había exportado a Hong Kong. ${ }^{12-14}$ Dentro de los contactos se encontraban tu- ristas procedentes de Toronto y de Singapur, quienes regresaron a sus ciudades de origen. Uno de los contactos a su vez contagió al menos 123 personas que desarrollaron la enfermedad dos semanas posterior a la exposición. ${ }^{15,16}$ El 15 marzo de 2003, la OMS nombró la enfermedad como SARS y la declaró una amenaza para la salud mundial. ${ }^{17,18}$ Posteriormente, el 22 de marzo a este nuevo beta coronavirus se le denominó SARS-CoV y se estableció como el causante de estas neumonías atípicas. ${ }^{12}$

Pocas semanas después, debido a la globalización y desplazamiento aéreo, el SARS-CoV se esparció a través de 29 países alrededor del mundo, principalmente en las ciudades de Toronto, Hong Kong, Singapur y Hanói. ${ }^{19}$ Las principales vías de trasmisión descritas fueron contacto cercano con personas infectadas con gotas respiratorias o fómites. ${ }^{20}$ El total de casos registrados fue de 8,096 y 774 muertes con una tasa de letalidad de $9.6 \%$. La OMS declaró el fin de la epidemia en julio 2003. ${ }^{17}$ Desde esta fecha a la actualidad, se han reportado brotes de SARS-CoV en cuatro ocasiones, tres de ellas fueron atribuidas a fallas en la bioseguridad en laboratorios de Singapur y Beijing. El cuarto incidente fue en la provincia de Guangdong, China, que dio como resultado cuatro casos esporádicos adquiridos en la comunidad durante un periodo de 6 semanas desde diciembre de 2003 hasta enero de 2004. Tres casos habían sido expuestos a animales (civeta de las palmeras) o fuentes ambientales, afortunadamente no hubo transmisión comunitaria. .,21,22 $^{2}$

EI SARS-CoV tiene como hospedero natural a los murciélagos; ${ }^{23-30}$ sin embargo, existen otros tipos de coronavirus como HCoV-OC43 y HKU1, cuyos hospederos son los roedores. ${ }^{31,32}$ En 2005, dos equipos de investigadores de manera independiente reportaron el nuevo coronavirus SARS-CoV en murciélagos herradura (genus Rhinolophus), con similitud en la secuencia de nucleótidos del 88-92\% a los SARS-CoV de humanos y de las civetas. ${ }^{29,33}$ Asimismo, varios coronavirus relacionados con SARS-CoV (SARSr-CoV) están ampliamente distribuidos y son prevalentes entre los murciélagos, que son sus hospederos naturales. ${ }^{5}$ No se ha encontrado progenitor directo del SARS-CoV en la población de murciélagos más allá de 15 años, lo que aunado al hecho de que la recombinación de RNA es muy frecuente, hace pensar que el surgimiento del SARS-CoV sea reciente. ${ }^{21,34-36} \mathrm{El}$ análisis de recombinación genética fuertemente soporta la hipótesis de que el SARS-CoV cepa SZ3 del mamífero civeta (de granja) se recombinó con dos cepas de murciélago ya 
existentes, las cepas WIV16, Rf4092 y de otras dos cepas prevalentes de SARSr-CoV, por medio de vía de trasmisión fecal-oral. ${ }^{24}$ Las civetas infectadas fueron trasportadas al mercado de Guangdong, donde el virus se diseminó entre las civetas del mercado y adquirió otras mutaciones antes de ser transferido al ser humano. ${ }^{5}$

\section{MERS-CoV}

En junio del 2012 se reportó la primera muerte por otro nuevo coronavirus, en Jeddah, Arabia Saudita. ${ }^{37,38} \mathrm{El}$ primer caso desarrolló neumonía y falleció por falla orgánica múltiple. ${ }^{39,40}$ Esta infección se ligó a un brote previo que sucedió en un hospital en Zarqa, Jordan en abril de 2012.41,42 Inicialmente, el virus se llamó coronavirus-EMC; sin embargo, por consenso internacional se nombró como MERS-CoV. ${ }^{43}$ En 2013 se registró un brote en Al Hasa, Arabia Saudita, que se asoció a pobres medidas de seguridad por parte del personal de salud. ${ }^{44}$ En 2015 ocurrió un brote en Corea del Sur, fue el segundo brote más grande después de Arabia Saudita, con 186 casos. ${ }^{45,46}$ En junio de 2017 se reportó otro brote de 34 casos, 17 de estos en personal de salud, en un hospital de Riyadh, Arabia Saudita. ${ }^{47}$

De abril de 2012 a diciembre de 2019 se han registrado 2,499 casos confirmados, 858 muertes, con tasa de letalidad del $34.3 \%$ en 27 países a nivel mundial. El país más afectado fue Arabia Saudita con 2,106 casos y 780 muertes.

EI MERS-CoV también tiene como hospedero natural a los murciélagos. ${ }^{27}$ Los casos de MERS-CoV estuvieron asociados a trasmisión con camellos dromedarios. ${ }^{37,40,41,48-50}$ La infección en camellos está documentada desde $1983 .^{50}$ La cepa MERSr-CoV Neoromicia/5038 (GenBank No. MF593268) aislada en Sudáfrica es la cepa más cercana filogenéticamente del MERS-CoV. La secuencia genómica del MERS-CoV del camello es idéntica en el $99 \%$ al del MERS-CoV humano. ${ }^{48} \mathrm{~A}$ diferencia del SARS-CoV, el MERS-CoV aún continúa en circulación causando brotes episódicos con un potencial riesgo epidémico global. ${ }^{51}$

\section{SADS-CoV}

Un brote de SADS (Swine Acute Diarrhoea Syndrome) fue identificado del 28 de octubre del 2016 al 2 de mayo de 2017. Se observó en cuatro granjas de cría de cerdos en la provincia de Guangdong, China, con mortalidad de hasta el $90 \%$ en las crías de los cerdos. Un nuevo coronavirus, el HKU2, originado en murcié- lagos, fue identificado como el agente causante. ${ }^{52,53}$ Los SADS-CoV aislados en cuatro cerdos de las granjas tenían semejanza en la secuencia genómica en 95\% con el coronavirus HKU2 de murciélago. ${ }^{54}$

\section{SARS-CoV-2}

El nuevo coronavirus llamado SARS-CoV-2 es el causante de la enfermedad COVID-19. Se reportaron los primeros casos en la ciudad de Wuhan, localizada en el departamento de Hubei, China a finales de diciembre del 2019. ${ }^{55}$ Una de las primeras advertencias del inicio de un brote epidémico en Wuhan fue por parte de un médico oftalmólogo, Li Wenliang, quien fue acusado de esparcir falsos rumores por parte del gobierno de China, finalmente falleció por neumonía severa por SARS-CoV-2 el 30 de diciembre de 2019. ${ }^{56}$ Se han reportado hasta la fecha más de 800,000 casos de COVID-19 en este país. Desde entonces, la propagación de la enfermedad ha afectado a todos los continentes, exceptuando a la Antártida. La OMS la declaró emergencia de salud pública de importancia internacional el 30 de enero y se categorizó como pandemia el 11 de marzo de $2020 . .^{57}$ En México, el primer caso fue diagnosticado el 28 de febrero de 2020.58

Un estudio epidemiológico en Wuhan al inicio de la pandemia relacionó a los pacientes infectados con visitas o con el contacto con personas que trabajaban en un mercado de mariscos y de venta de animales exóticos. ${ }^{59}$ Sin embargo, para la progresión de la pandemia, la trasmisión persona a persona fue el medio principal. Se ha encontrado una alta carga del virus en orofaringe, incluso en etapas tempranas de la enfermedad en pacientes con síntomas leves, por ello, la vía principal de contagio de SARS-CoV-2 es por gotas respiratorias, que son trasmitidas cuando una persona infectada tose, estornuda o habla y estas gotas entran en contacto directo con boca, ojos y nariz de otra persona. ${ }^{60}$ Otro mecanismo de trasmisión es el contacto con superficies infectadas y posterior contacto con mucosas. ${ }^{61}$ Las gotas respiratorias no viajan más de 2 metros y no se quedan suspendidas en el aire; sin embargo, van Doremalen y colaboradores reportaron de manera experimental que el SARS-CoV-2 podría permanecer en el aire viable hasta por tres horas. ${ }^{62} \mathrm{El}$ RNA de SARS-CoV-2 ha sido aislado de muestras de sangre $y$ de heces, ${ }^{63-65}$ y se han cultivado virus vivos de muestra de heces; ${ }^{66}$ sin embargo, esta vía de trasmisión no es importante. ${ }^{67}$ El periodo de incubación se ha estimado en 5-6 días, con un rango de uno a 14 días. ${ }^{68,69}$ 


\section{Epidemiología de la infección por SARS-CoV-2}

Para el 27 de mayo de 2020 se han reportado $5,488,825$ casos confirmados de SARS-CoV-2 en todo el mundo, el continente más afectado es el americano con 2,495,924 casos, seguido de Europa con $2,061,828$ casos (Figura 1 y Tabla 1). ${ }^{70,71}$

El Centro de Control y Prevención de Enfermedades en China reportó inicialmente una serie de casos donde se estudiaron 72,314 pacientes, donde 44,672 $(61.8 \%)$ casos fueron confirmados por reacción en cadena de la polimerasa (PCR, por sus siglas en inglés) con hisopado nasofaríngeo, 16,186 (22.4\%) casos fueron sospechosos, 10,567 (14.6\%) casos fueron sintomáticos y $889(1.2 \%)$ casos fueron asintomáticos, $2,087(4.7 \%)$ pacientes se catalogaron como en estado crítico con necesidad de ventilación mecánica y $1,023(2.3 \%)$ pacientes fallecieron. ${ }^{72}$

En otro estudio multicéntrico de Guan y colaboradores en China, de 1,099 pacientes confirmados con SARS-CoV-2, el $6.1 \%$ requirieron ingreso a la Unidad de Cuidados Intensivos (UCI), 2.3\% necesitó ventilación mecánica asistida (VMA) y $1.4 \%$ falleció. ${ }^{73} \mathrm{Wu}$ y su equipo informaron en una cohorte de un hospital de Wuhan, China, 201 pacientes con diagnóstico de neumonía severa por COVID-19, 84 pacientes (41.8\%) desarrollaron síndrome de dificultad respiratoria aguda (ARDS, por sus siglas en inglés de Acute Respiratory Distress Syndrome) y de estos 44/84 pacientes $(52.4 \%)$ fallecieron. Los factores de riesgo para muerte fueron la edad avanzada (hazard ratio [HR], 6.17; con intervalo de confianza al 95\% [IC 95\%] 3.26-11.67) y elevación de lactato deshidrogenasa (HR 1.30; IC 95\%, 1.11-1.52). ${ }^{74}$ Para el 19 de marzo de 2020 en la región de Lombardía, Italia, se reportaron 19,884 casos confirmados de COVID-19, de estos 1,006 pacientes $(5 \%)$ requirieron $\mathrm{VMI}$ y 2,168 (10.9\%) pacientes murieron.

El 20 de enero de 2020 se reportó el primer caso de COVID-19 en Estados Unidos de América (EUA). ${ }^{75}$ Bhatraju y su grupo en Seattle, EUA, reportaron una serie de 24 pacientes de nueve terapias intensivas con pacientes con ARDS por SARS-CoV-2, 18/28 pacientes $(75 \%)$ requirieron $\mathrm{VMI}, 17 / 24$ pacientes $(70.8 \%)$ se le administraron vasopresores, $12 / 24$ pacientes (50\%) murieron entre el día uno y 18 del seguimiento en la UCl. ${ }^{76}$ Richardson y colegas, en Nueva York, EUA, publicaron una cohorte de 5,700 pacientes hospitalizados por COVID-19, el $14.2 \%$ fueron tratados en la UCI, el $12.2 \%$ requirieron VMA, el $3.2 \%$ utilizaron terapia de reemplazo renal (TRR) y el $21 \%$ fallecieron. ${ }^{77}$

Los primeros tres casos en México fueron reportados el 28 de febrero de 2020, un caso en el estado de Sinaloa y dos casos en la Ciudad de México. Para el 27 de mayo de 2020, en México se han reportado 78,023 casos confirmados, prevalencia de 61.1 casos por cada 100,000 habitantes, incidencia de 12.2 casos por cada 100,000 habitantes, 33,566 casos sospechosos y 8,597 defunciones (letalidad del 9.07\%). De los casos confirmados, se hospitalizó al $36.20 \%$ y se recuperaron de

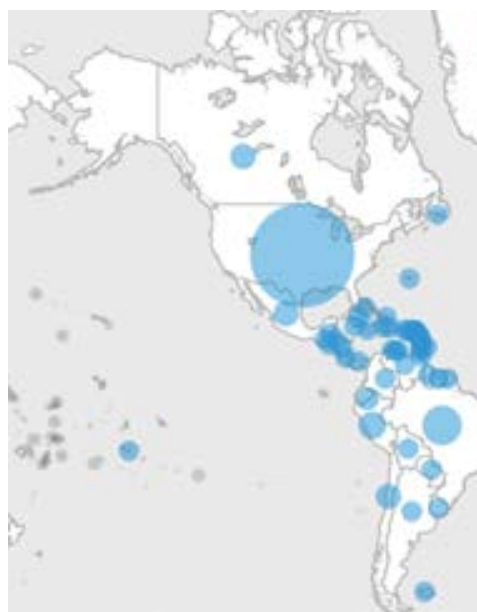

Casos nuevos: 99,024
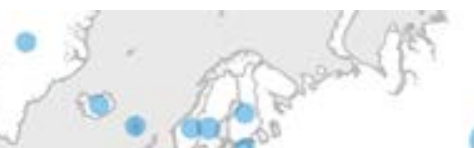

siving
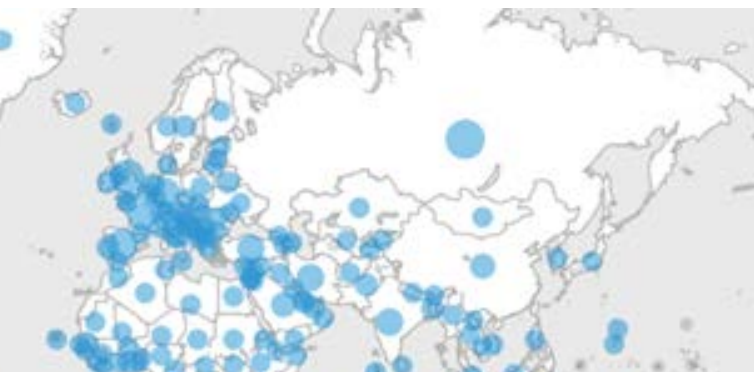

.

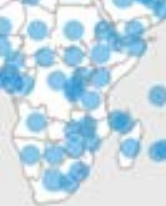

El tamaño del círculo está en relación a la cantidad de casos
Casos confirmados: $5,488,825$
Muertes: 349,095
Fuente: OMS

27/mayo/2020
Figura 1:

Distribución mundial de casos confirmados de SARS-CoV-2 por país. Modificado de: https://covid19.who.int/ Última actualización: 27/mayo/2020. ${ }^{71}$ 
Tabla 1: Distribución mundial de casos confirmados y muertes por SARS-CoV-2.

\begin{tabular}{lcr}
\hline \multicolumn{1}{c}{ Región } & Casos confirmados & Muertes \\
\hline Global & $5,488,825$ & 349,095 \\
América & $2,495,924$ & 145,810 \\
Europa & $2,061,828$ & 176,226 \\
Mediterráneo oriental & 449,590 & 11,452 \\
Pacífico oeste & 176,404 & 6,927 \\
Sudeste de Asia & 218,523 & 6,359 \\
África & 85,815 & 2,308 \\
\hline
\end{tabular}

Datos actualizados al 27 de mayo de 2020.70

la enfermedad 54,383 pacientes. ${ }^{78}$ Bello-Chavolla y colaboradores reportaron en México (27 abril) una cohorte con 15,529 pacientes con COVID-19, de los cuales 6,042 pacientes $(38.9 \%)$ se hospitalizaron, 4,588 (29.5\%) presentaron cuadro de neumonía y 676 (4.4\%) requirieron ingreso a la $\mathrm{UCl}$ con apoyo VMA en 669 de los casos (4.3\%). La muerte ocurrió en 1,434 pacientes $(9.2 \%)$, los factores de riesgo asociados con mayor mortalidad fueron obesidad (HR 1.567, IC 95\% 1.2731.928), inicio de diabetes mellitus antes de los 40 años de edad (HR 2.764, 1.718-4.447), embarazo (HR 2.62, 1.08-6.35), edad mayor a 65 años (HR 2.12, 1.87-2.41) y cuadro clínico de neumonía (HR 5.46, 4.74-6.29). ${ }^{79}$

Mejía y su grupo, en un hospital de la Ciudad de México, reportaron una cohorte de 329 pacientes con COVID-19, de los cuales 115 pacientes requirieron ingreso a la $\mathrm{UCl}(34.9 \%)$, al momento de finalizar el estudio $61 / 115$ (53\%) de los pacientes de UCI fallecieron, $25 / 115$ (22\%) fueron dados de alta y $29 / 115$ (25\%) permanecieron hospitalizados. ${ }^{80}$

\section{COVID-19 en pacientes con}

enfermedad renal crónica

El impacto del SARS-CoV-2 en pacientes con enfermedad renal crónica (ERC) ha sido escasamente reportada. Los primeros reportes en unidades de HD en Wuhan, China (Ma y colaboradores, y Wang y su equippo) describieron a pacientes en HD crónica y enfermedad por COVID-19 con menor severidad y mortalidad. ${ }^{81,82}$ Otro estudio de 65 unidades de HD en Wuhan, China, del 1 de enero al 10 de marzo de 2020, analizó los datos de 131 pacientes, de los cuales 101 pacientes (77\%) tuvieron enfermedad leve-moderada, 30 pacientes (23\%) enfermedad severa y 16 pacientes ARDS. ${ }^{83}$

En una investigación de Wu y su grupo, en el Hospital de Tongren de la universidad de Wuhan, se in- cluyeron 49 pacientes en HD crónica hospitalizados por COVID-19 y se compararon con 52 controles sin enfermedad renal. Los pacientes en HD presentaron mayor cantidad de complicaciones como choque (16 vs $4 \%$ ), ARDS (20 vs $6 \%$ ), arritmias (18 vs $2 \%$ ) y daño cardiaco agudo (29 vs $8 \%$ ). En el grupo de HD $25 \%$ necesitaron $\mathrm{VMNI}$ comparado con $6 \%$ en el grupo control; el $6 \%$ requirieron de $\mathrm{VMI}$ en el grupo de HD vs $2 \%$ del grupo control. Murió el $14 \%$ del grupo de HD y $4 \%$ en el grupo control. ${ }^{84}$

La experiencia en Europa fue reportada por Goicoechea y colaboradores en España (50\% de sus pacientes desarrollaron ARDS), ${ }^{85}$ Scarpioni y colegas en Italia ${ }^{86}$ y La Milia y su equipo en la región de Lombardía, Italia. Este último reportó a 33/188 pacientes asintomáticos que resultaron positivos para la prueba de SARS-CoV-2 (Tabla 2). ${ }^{87}$

\section{COVID-19 en pacientes con trasplante renal}

En la población de trasplante de riñón la información es menor. El uso de tratamiento inmunosupresor en estos pacientes podría modificar el curso clínico de la infección. ${ }^{88}$ Existen antecedentes de infección en pacientes trasplantados con coronavirus en epidemias previas. Kumar y colaboradores reportaron en 2003 en Toronto un caso fatal de SARS-CoV en un paciente trasplantado de hígado, su cuadro clínico fue similar a los no trasplantados. ${ }^{89}$ Alghamdi y su grupo en 2015 reportaron dos casos de trasplante renal con infección con MERS-CoV, la presentación fue atípica en uno de los pacientes, sin fiebre y dificultad respiratoria, el otro paciente, con un mes postrasplante, presentó enfermedad leve y se mantuvo estable a pesar de haber tenido inducción con timoglobulina. El desenlace para el primer paciente fue fatal y el último se recuperó de manera satisfactoria. ${ }^{90}$

El primer caso reportado en esta pandemia por SARS-CoV-2 sucedió en Wuhan, China, y tuvo las mismas características que los pacientes no trasplantados y su evolución fue adecuada. ${ }^{91}$

Zhang y colaboradores reportaron la primera serie de cinco pacientes trasplantados y COVID-19 en la ciudad de Wuhan, China. Todos fueron receptores de donante fallecido, el $100 \%$ presentaron fiebre y tos. Todos los pacientes con saturación $\geq 96 \%$, ninguno de los pacientes requirió de VMI ni falleció. ${ }^{92}$

Zhu y su grupo informaron una serie de 10 pacientes receptores de trasplante renal y COVID-19 también en la ciudad de Wuhan, China, 7/10 pacientes eran receptores de trasplante renal de donante falleci- 
do, $9 / 10$ casos presentaron fiebre $\geq 38^{\circ} \mathrm{C}, 3 / 10$ casos utilizaron VMA y $5 / 10$ casos se clasificaron como severos. Al final del estudio $8 / 10$ pacientes se egresaron del hospital, $1 / 8$ permanecía internado pero estable y $1 / 10$ falleció. $^{93}$

Dos casos en receptores de trasplante renal en Corea del Sur fueron reportados con buena evolución y sin necesidad de VMA. ${ }^{94}$

Gandolfini y su equipo reportaron en Parma, Italia, dos casos de COVID-19 en pacientes trasplantados de donante fallecido, ambos pacientes requirieron de VMA, uno desarrolló lesión renal aguda (LRA) y murió a los cinco días posteriores. ${ }^{95}$

En Barcelona, España, se reportó el primer caso de COVID-19 en un RTR de donante fallecido el 20 de marzo del 2020, desarrolló ARDS y LRA, a los 10 días de seguimiento requirió VMA ${ }^{96}$ Fernández-Ruiz y colegas en el hospital 12 de octubre de Madrid, España, reportaron una cohorte de 18 pacientes receptores de trasplante de órgano sólido, riñón en 8/18 casos (44.4\%), hígado en $6 / 18$ casos $(33.3 \%$ ) y corazón en $4 / 18$ casos $(22.2 \%)$. Murieron $5 / 18$ pacientes $(27.8 \%)$, $7 / 18(38.9 \%)$ desarrollaron ARDS y la necesidad de ingreso a la UCI en $11.1 \%$. En el seguimiento a 18 días, 13 pacientes habían sobrevivido, de los cuales $4 / 13$ (30.8\%) desarrollaron ARDS, 1/13 (7.7\%) permaneció en condición estable y $8 / 13(61.5 \%)$ se egresaron a casa. ${ }^{97}$

Banerjee y colaboradores en Londres, Inglaterra, reportaron una serie de siete casos de RTR, 3/7 pacientes estaban dentro de tres meses del trasplante, $4 / 7$ ingresaron a $\mathrm{UCI}, 1 / 7$ falleció. ${ }^{98}$ Alberici y su equipo en Brescia, Italia, reportaron una cohorte de 20 pacientes RTR con neumonía por SARS-CoV-2. El 30\% desarrolló LRA y requirieron de $\mathrm{HD}$, el $20 \%$ ingresó a la $\mathrm{UCI}$, el $50 \%$ necesitaron VMNI y $5 / 20$ pacientes (25\%) murieron en el seguimiento, la mediana de días de seguimiento fue de 15 con rango intercuartil (RIC) de 15-19 días. ${ }^{99}$

Tschopp y su equipo del Swiss Transplant Cohort Study (STCS) reportaron un total de 21 pacientes trasplantados en Suiza, que se distribuyeron en 10/21 casos de riñón, 5/21 casos de hígado, 1/21 caso de páncreas, $1 / 21$ caso pulmón, 1/21 caso de corazón y $3 / 21$ casos de trasplante combinado. De ellos, 20/21 pacientes $(95.2 \%)$ se hospitalizaron, de éstos, cinco requirieron ingreso a la UCl y cuatro necesitaron VMA. Las complicaciones médicas más frecuentes fueron LRA en 9/21 (43\%), ARDS 4/21 (19\%), choque séptico $2 / 21(10 \%)$ y $1 / 21(4.7 \%)$ tromboembolia pulmonar. En una mediana de seguimiento a 33 días 16/21 (76.2\%) se egresaron a casa, $3 / 21$ (14.2\%) permanecieron hospitalizados y $2 / 21(9.5 \%)$ fallecieron. ${ }^{100}$

Abrishami y colaboradores reportaron una serie de 12 casos de pacientes receptores de trasplante renal en Terán, Irán. El 83.3\% ingresaron a la UCI, de los cuales $9 / 10$ fueron intubados y ocho pacientes fallecieron que representaban el $66.6 \%$ del total. ${ }^{101}$

Akalin y su grupo en la ciudad de Nueva York, EUA, reportaron una serie de 36 pacientes RTR, 14/36 $(39 \%)$ raza negra y $15 / 36(42 \%)$ raza hispana, $27 / 36$ (75\%) receptores de donante fallecido. El 39\% requirieron de VMA, 21\% necesitaron TRR. En un seguimiento con mediana de 21 días (RIC 14-28 días), 10 pacientes murieron, que representaban el $28 \%(10 / 36)$ del total. ${ }^{102}$

Nair y colegas reportaron en la ciudad de Nueva York, EUA, una cohorte de 10 pacientes RTR con prueba positiva para SARS-CoV-2, 9/10 casos fueron hospitalizados y $5 / 9$ fueron ingresados a la $\mathrm{UCl}, 3 / 9$ requirieron intubación y fallecieron. ${ }^{103}$

El programa de trasplante de la Universidad de Columbia en Nueva York publicó una serie de 15 pacientes de receptores de trasplante renal $(80 \%$ donante

Tabla 2: Principales características de los estudios de COVID-19 en enfermedad renal crónica.

\begin{tabular}{|c|c|c|c|c|c|c|c|}
\hline $\mathrm{Ma}^{81}$ Wuhan, China & 230 & $37(16)$ & 100 & ND & ND & ND & $6(16.2)$ \\
\hline Wang ${ }^{82}$ Wuhan, China & 201 & $5(2.4)$ & 100 & 0 & 0 & 0 & $0(0)$ \\
\hline Xiong ${ }^{83}$ Wuhan, China & 7,514 & $131(1.7)$ & 100 & $36(27)$ & $23(21)$ & $2(1.8)$ & ND \\
\hline Wu ${ }^{84}$ Wuhan, China & NA & 49 (NA) & 100 & $12(25)$ & $12(25)$ & $3(6)$ & $7(14)$ \\
\hline Goicoechea ${ }^{85}$ Madrid, España & 282 & $36(12.7)$ & 100 & ND & $12(33)$ & ND & $11(30.5)$ \\
\hline
\end{tabular}

CRRT = terapia reemplazo renal lenta continua, $I H$ = ingreso hospitalario, $P H D=$ población en unidad de hemodiálisis, VMNI = ventilación mecánica no invasiva, VMI = ventilación mecánica invasiva, $N A$ = no aplica, $N D$ = datos no disponibles. * La mortalidad es calculada con respecto a la población total en la unidad de HD. ** La mortalidad fue calculada con respecto a la población que se ingresó a hospitalización (13/25). 
Tabla 3: Principales características de los estudios de COVID-19 en receptores de trasplante renal.

\begin{tabular}{|c|c|c|c|c|c|c|c|}
\hline Autor/país & $\mathrm{n}$ & $\begin{array}{c}\mathbb{H} \\
n(\%)\end{array}$ & $\begin{array}{l}\text { UCl } \\
n(\%)\end{array}$ & $\begin{array}{l}\text { LRA } \\
\mathrm{n}(\%)\end{array}$ & $\begin{array}{l}\text { VMNI } \\
\mathrm{n}(\%)\end{array}$ & $\begin{array}{l}\text { VMI } \\
n(\%)\end{array}$ & $\begin{array}{c}\text { Mortalidad } \\
n(\%)\end{array}$ \\
\hline Zhang ${ }^{92}$ Wuhan, China & 5 & $5(100)$ & 0 & ND & 0 & 0 & 0 \\
\hline Gandolfini95 Parma, Italia & 2 & $2(100)$ & $2(100)$ & $1(50)$ & $1(50)$ & $1(50)$ & $1(50)$ \\
\hline Fernández-Ruiz ${ }^{97}$ Madrid, España & 18 & $18(100)$ & $2(11)$ & ND & $3(17)$ & $2(11)$ & $5(28)$ \\
\hline Banerjee ${ }^{98}$ Londres, UK & 7 & $7(100)$ & $4(57)$ & $4(57)$ & $2(28.5)$ & $2(28.5)$ & $1(14)$ \\
\hline Abrishami' ${ }^{101}$ Terán, Irán & 12 & $12(100)$ & $10(83)$ & $6(50)$ & $3(25)$ & $9(75)$ & $8(66.6)$ \\
\hline Akalin'102 Nueva York, EUA & 36 & $28(78)$ & ND & $6(21)^{\star \star *}$ & $\mathrm{ND}$ & $11(39)$ & $10(28)$ \\
\hline Nair ${ }^{103}$ Nueva York, EUA & 10 & $9(90)$ & $5(55)$ & $4(44)$ & ND & $3(33)$ & $3(33)$ \\
\hline Mohan ${ }^{104}$ Nueva York, EUA & 15 & $15(100)$ & ND & $6(40)$ & ND & $4(27)$ & $2(13)$ \\
\hline Pereira ${ }^{105}$ Nueva York, EUA & 90 & $68(76)$ & $23(34)$ & ND & $10(12)$ & $24(35)$ & $16(18)$ \\
\hline
\end{tabular}

$\mathrm{HH}$ = ingreso hospitalario, $\mathrm{PHD}=$ población en unidad de hemodialisis, VMNI = ventilación mecánica no invasiva, VMI = ventilación mecánica invasiva, $\mathrm{NA}=$ no aplica, $\mathrm{ND}$ = datos no disponibles, EUA = Estados Unidos de América, UK = Reino Unido. * El estudio no especifica modalidad de ventilación mecánica. ${ }^{* *}$ La mortalidad es calculada con respecto a la n total. ${ }^{\star * \star}$ Seis pacientes requirieron terapia de reemplazo renal.

fallecido) y enfermedad por COVID-19 que requirieron de hospitalización, otros ocho pacientes con prueba positiva para SARS-CoV-2 y sintomatología leve que se manejaron de manera ambulatoria no se incluyeron en el análisis. El $40 \%$ desarrollaron LRA, de éstos, dos pacientes requirieron TRR. El 27\% necesitaron de VMA, fallecieron $2 / 15$ casos $(13 \%)$ y se egresaron a casa 8/15 (53\%). Uno de los pacientes desarrolló síntomas mientras era tratado para rechazo activo mediado por anticuerpos con altas dosis de esteroide y plasmaféresis, las cuales fueron suspendidas, el otro paciente desarrolló síntomas al terminar el curso de inducción con timoglobulina posterior a la cirugía de trasplante renal de donante fallecido. ${ }^{104}$

Hasta ahora, la serie más grande reportada en EUA es de Pereira y colaboradores en Nueva York con 90 pacientes receptores de trasplante de órgano sólido, $46 / 90(51.1 \%)$ fueron de riñón, $17 / 90(18.8 \%)$ casos de pulmón, 13/90 (14.4\%) casos de hígado, 9/90 (10\%) casos de corazón, $5 / 90(5.5 \%)$ casos de trasplantes duales. El $75.5 \%$ requirió de hospitalización, de los cuales 23/68 (34\%) necesitó ingreso a la UCl, 10/68 $(12 \%)$ requirieron VMNI y 24/68 (35.2\%) requirieron de intubación. Fallecieron 16 pacientes (18\%). El 54.4\% se egresó a casa. ${ }^{105}$

El primer caso de COVID-19 en un paciente trasplantado dual (hígado y riñón) en Brasil se reportó el 4 de mayo, con un cuadro de fiebre y diarrea, no hubo necesidad de ventilación mecánica invasiva y su evolución fue satisfactoria (Tabla 3). ${ }^{106}$

En México el Centro Nacional de Trasplantes (CENATRA) emitió un boletín informativo especial de Trasplantes y COVID-19 actualizado al 25 de mayo de
2020 , donde se ha reportado un acumulado de 28 casos confirmados (26 de riñón y dos de hígado), ocho casos negativos y tres casos sospechosos, además ocho receptores de trasplante fallecidos con COVID-19 confirmado. La entidad federativa con mayor cantidad de casos es la Ciudad de México con 22, seguida de Jalisco con tres, Puebla con dos y Coahuila un caso. ${ }^{107}$

\section{CONCLUSIÓN}

Esta generación de seres humanos quedará marcada por una de las pandemias más graves de la historia. El SARS-CoV-2 es un virus altamente contagioso, puede sobrevivir en varias superficies por periodos prolongados de tiempo, lo que aumenta su diseminación. Los pacientes con enfermedad renal crónica y receptores de trasplante renal son población con mayor probabilidad de complicaciones por el SARS-CoV-2 incluyendo la mayor posibilidad de muerte.

\section{REFERENCIAS}

1. Kahn JS, McIntosh K. History and recent advances in coronavirus discovery. Pediatr Infect Dis J. 2005; 24 (11 Suppl): S223-S226.

2. Tyrrell DAJ, Bynoe ML. Cultivation of viruses from a high proportion of patients with colds. Lancet. 1966; 287 (7428): 76-77.

3. Almeida JD, Tyrrell DAJ. The morphology of three previously uncharacterized human respiratory viruses that grow in organ culture. J Gen Virol. 1967; 1 (2): 175-178.

4. Tyrrell DAJ, Almeida JD, Cunningham $\mathrm{CH}$, Dowdle WR, Hofstad MS, Mclntosh $\mathrm{K}$ et al. Coronaviridae. Intervirology. 1975; 5 (1-2): 76-82.

5. Cui J, Li F, Shi ZL. Origin and evolution of pathogenic coronaviruses. Nat Rev Microbiol. 2019; 17 (3): 181-192. 
6. Hui DSC, Zumla A. Severe acute respiratory syndrome. Infect Dis Clin N Am. 2019; 33 (4): 869-889.

7. Zhong N, Zheng B, Li Y, Poon L, Xie Z, Chan $\mathrm{K}$ et al. Epidemiology and cause of severe acute respiratory syndrome (SARS) in Guangdong, People's Republic of China, in February, 2003. Lancet. 2003; 362 (9393): 1353-1358.

8. Drosten C, Günther S, Preiser W, van der Werf S, Brodt HR, Becker $S$ et al. Identification of a novel coronavirus in patients with severe acute respiratory syndrome. N Engl J Med. 2003; 348 (20): 1967-1976.

9. Fouchier RAM, Kuiken T, Schutten M, van Amerongen G, van Doornum GJJ, van den Hoogen BG et al. Koch's postulates fulfilled for SARS virus. Nature. 2003; 423 (6937): 240-240.

10. Ksiazek TG, Erdman D, Goldsmith CS, Zaki SR, Peret T, Emery $S$ et al. A novel coronavirus associated with severe acute respiratory syndrome. N Engl J Med. 2003; 348 (20): 1953-1966.

11. Zhao Z, Zhang F, Xu M, Huang K, Zhong W, Cai W et al. Description and clinical treatment of an early outbreak of severe acute respiratory syndrome (SARS) in Guangzhou, PR China. J Med Microbiol. 2003; 52 (Pt 8): 715-720.

12. Peiris JSM, Lai ST, Poon LLM, Guan Y, Yam LYC, Lim W et al. Coronavirus as a possible cause of severe acute respiratory syndrome. Lancet (London, England). 2003; 361 (9366): 13191325.

13. Tsang KW, Ho PL, Ooi GC, Yee WK, Wang T, Chan-Yeung M et al. A cluster of cases of severe acute respiratory syndrome in Hong Kong. N Engl J Med. 2003; 348 (20): 1977-1985.

14. Nicholls JM, Poon LL, Lee KC, Ng WF, Lai ST, Leung CY et al. Lung pathology of fatal severe acute respiratory syndrome. Lancet. 2003; 361 (9371): 1773-1778.

15. Wong RSM, Hui DS. Index patient and SARS outbreak in Hong Kong. Emerg Infect Dis. 2004; 10 (2): 339-341.

16. Lee N, Hui D, Wu A, Chan P, Cameron P, Joynt GM et al. A major outbreak of severe acute respiratory syndrome in Hong Kong. N Engl J Med. 2003; 348 (20): 1986-1994.

17. WHO. Summary of probable SARS cases with onset of illness from 1 November 2002 to 31 July 2003 [Internet]. 2003 [cited 2020 Apr 18]. Available in: https://www.who.int/csr/sars/ country/table2004_04_21/en/

18. WHO. Update 95-SARS: Chronology of a serial killer [Internet]. Available in: https://www.who.int/csr/don/2003_07_04/en/

19. Hui DSC, Sung JJY. Severe acute respiratory syndrome. Chest. 2003; 124 (1): 12-15.

20. Peiris JSM, Yuen KY, Osterhaus ADME, Stöhr K. The severe acute respiratory syndrome. N Engl J Med. 2003; 349 (25): 2431-2441.

21. Song HD, Tu CC, Zhang GW, Wang SY, Zheng K, Lei LC et al. Cross-host evolution of severe acute respiratory syndrome coronavirus in palm civet and human. Proc Natl Acad Sci. 2005; 102 (7): 2430-2435.

22. Wang M, Yan M, Xu H, Liang W, Kan B, Zheng B et al. SARSCoV infection in a restaurant from palm civet. Emerg Infect Dis. 2005; 11 (12): 1860-1865.

23. Lau SKP, Li KSM, Tsang AKL, Lam CSF, Ahmed S, Chen H et al. Genetic characterization of Betacoronavirus lineage $C$ viruses in bats reveals marked sequence divergence in the spike protein of pipistrellus bat coronavirus HKU5 in Japanese pipistrelle: implications for the origin of the novel Middle East respiratory syndrome coronavirus. J Virol. 2013; 87 (15): 86388650.

24. Hu B, Zeng LP, Yang XL, Ge XY, Zhang W, Li B et al. Discovery of a rich gene pool of bat SARS-related coronaviruses provides new insights into the origin of SARS coronavirus. PLoS Pathog. 2017; 13 (11): e1006698.

25. Yang XL, Hu B, Wang B, Wang MN, Zhang Q, Zhang W et al. Isolation and characterization of a novel bat coronavirus closely related to the direct progenitor of severe acute respiratory syndrome coronavirus. J Virol. 2016; 90 (6): 3253-3256.

26. Ge XY, Li JL, Yang XL, Chmura AA, Zhu G, Epstein JH et al. Isolation and characterization of a bat SARS-like coronavirus that uses the ACE2 receptor. Nature. 2013; 503 (7477): 535538.

27. Ithete NL, Stoffberg S, Corman VM, Cottontail VM, Richards LR, Schoeman MC et al. Close relative of human Middle East respiratory syndrome coronavirus in bat, South Africa. Emerg Infect Dis. 2013; 19 (10): 1697-1699.

28. Kan B, Wang M, Jing $\mathrm{H}$, Xu H, Jiang $\mathrm{X}$, Yan $\mathrm{M}$ et al. Molecular evolution analysis and geographic investigation of severe acute respiratory syndrome coronavirus-like virus in palm civets at an animal market and on farms. J Virol. 2005; 79 (18): 11892-11900.

29. Lau SKP, Woo PCY, Li KSM, Huang Y, Tsoi HW, Wong BHL et al. Severe acute respiratory syndrome coronavirus-like virus in Chinese horseshoe bats. Proc Natl Acad Sci. 2005; 102 (39): 14040-14045.

30. Guan Y, Zheng BJ, He YQ, Liu XL, Zhuang ZX, Cheung CL et al. Isolation and characterization of viruses related to the SARS coronavirus from animals in southern China. Science (New York, NY). 2003; 302 (5643): 276-278.

31. Su S, Wong G, Shi W, Liu J, Lai ACK, Zhou J et al. Epidemiology, genetic recombination, and pathogenesis of coronaviruses. Trends Microbiol. 2016; 24 (6): 490-502.

32. Forni D, Cagliani R, Clerici M, Sironi M. Molecular evolution of human coronavirus genomes. Trends Microbiol. 2017; 25 (1): 35-48.

33. Li W, Shi Z, Yu M, Ren W, Smith C, Epstein JH et al. Bats are natural reservoirs of SARS-like coronaviruses. Science (New York, NY). 2005; 310 (5748): 676-679.

34. Hon CC, Lam TY, Shi ZL, Drummond AJ, Yip CW, Zeng F et al. Evidence of the recombinant origin of a bat severe acute respiratory syndrome (SARS)-like coronavirus and its implications on the direct ancestor of SARS coronavirus. J Virol. 2008; 82 (4): 1819-1826.

35. Zhao Z, Li H, Wu X, Zhong Y, Zhang K, Zhang YP et al. Moderate mutation rate in the SARS coronavirus genome and its implications. BMC Evol Biol. 2004; 4 (1): 21.

36. Lai MM, Cavanagh D. The molecular biology of coronaviruses. Adv Virus Res. 1997; 48: 1-100.

37. Azhar El, El-Kafrawy SA, Farraj SA, Hassan AM, Al-Saeed MS, Hashem AM et al. Evidence for camel-to-human transmission of MERS coronavirus. N Engl J Med. 2014; 370 (26): 24992505.

38. WHO. Middle East respiratory syndrome coronavirus (MERSCoV) [Internet]. [cited 2020 Apr 19]. Available in: https://www. who.int/emergencies/mers-cov/en/

39. Zaki AM, van Boheemen S, Bestebroer TM, Osterhaus ADME, Fouchier RAM. Isolation of a novel coronavirus from a man with pneumonia in Saudi Arabia. N Engl J Med. 2012; 367 (19): 1814-1820.

40. Memish ZA, Perlman S, van Kerkhove MD, Zumla A. Middle East respiratory syndrome. Lancet (London, England). 2020; 395 (10229): 1063-1077.

41. Hijawi B, Abdallat M, Sayaydeh A, Alqasrawi S, Haddadin $\mathrm{A}$, Jaarour $\mathrm{N}$ et al. Novel coronavirus infections in Jordan, April 2012: epidemiological findings from a retrospective 
investigation. East Mediterr Health J. 2013; 19 Suppl 1: S12-S18.

42. Al-Abdallat MM, Payne DC, Alqasrawi S, Rha B, Tohme RA, Abedi GR et al. Hospital-associated outbreak of middle east respiratory syndrome coronavirus: a serologic, epidemiologic, and clinical description. Clin Infect Dis. 2014; 59 (9): 1225-1233.

43. de Groot RJ, Baker SC, Baric RS, Brown CS, Drosten C, Enjuanes $L$ et al. Middle east respiratory syndrome coronavirus (MERS-CoV): announcement of the coronavirus study group. J Virol. 2013; 87 (14): 7790-7792.

44. Assiri A, McGeer A, Perl TM, Price CS, al Rabeeah AA, Cummings DAT et al. Hospital outbreak of middle east respiratory syndrome coronavirus. N Engl J Med. 2013; 369 (5): 407-416.

45. Oh M, Park WB, Park SW, Choe PG, Bang JH, Song KH et al. Middle East respiratory syndrome: what we learned from the 2015 outbreak in the Republic of Korea. Korean J Intern Med. 2018; 33 (2): 233-246.

46. Kim KH, Tandi TE, Choi JW, Moon JM, Kim MS. Middle east respiratory syndrome coronavirus (MERS-CoV) outbreak in South Korea, 2015: epidemiology, characteristics and public health implications. J Hosp Infect. 2017; 95 (2): 207-213.

47. Alanazi KH, Killerby ME, Biggs HM, Abedi GR, Jokhdar H, Alsharef AA et al. Scope and extent of healthcare-associated Middle East respiratory syndrome coronavirus transmission during two contemporaneous outbreaks in Riyadh, Saudi Arabia, 2017. Infect Control Hosp Epidemiol. 2019; 40 (1): 79-88.

48. Chu DKW, Hui KPY, Perera RAPM, Miguel E, Niemeyer D, Zhao $\mathrm{J}$ et al. MERS coronaviruses from camels in Africa exhibit region-dependent genetic diversity. Proc Natl Acad Sci U S A. 2018; 115 (12): 3144-3149.

49. Raj VS, Farag EABA, Reusken CBEM, Lamers MM, Pas $\mathrm{SD}$, Voermans $\mathrm{J}$ et al. Isolation of MERS coronavirus from a dromedary camel, Qatar, 2014. Emerg Infect Dis. 2014; 20 (8): 1339-1342.

50. Müller MA, Corman VM, Jores J, Meyer B, Younan M, Liljander A et al. MERS coronavirus neutralizing antibodies in camels, Eastern Africa, 1983-1997. Emerg Infect Dis. 2014; 20 (12): 2093-2095.

51. Zumla A, Hui DS, Perlman S. Middle East respiratory syndrome. Lancet (London, England). 2015; 386 (9997): 995-1007.

52. Gong L, Li J, Zhou Q, Xu Z, Chen L, Zhang Y et al. A New BatHKU2-like Coronavirus in Swine, China, 2017. Emerg Infect Dis. 2017; 23 (9): 1607-1609.

53. Zhou P, Fan H, Lan T, Yang XL, Shi WF, Zhang W et al. Fatal swine acute diarrhoea syndrome caused by an HKU2-related coronavirus of bat origin. Nature. 2018; 556 (7700): 255-258.

54. Lau SKP, Woo PCY, Li KSM, Huang Y, Wang M, Lam CSF et al. Complete genome sequence of bat coronavirus HKU2 from Chinese horseshoe bats revealed a much smaller spike gene with a different evolutionary lineage from the rest of the genome. Virology. 2007; 367 (2): 428-439.

55. Huang C, Wang Y, Li X, Ren L, Zhao J, Hu Y et al. Clinical features of patients infected with 2019 novel coronavirus in Wuhan, China. Lancet. 2020; 395 (10223): 497-506.

56. Green A. Li Wenliang. Lancet [Internet]. 2020; 395 (10225): 682. Available in: https://doi.org/10.1016/S0140-6736 (20)30382-2

57. WHO. This statement is updated on an ongoing basis, in response to evolving events and common media queries. [Internet]. 2020 [cited 2020 May 18]. Available in: www.who. int/news-room/detail/27-04-2020-who-timeline---covid-19

58. Secretaría de Salud. Información Internacional y Nacional sobre nuevo Coronavirus (COVID-2019) [Internet]. 2020 [citado 18 de mayo de 2020]. Disponible en: https://www. gob.mx/cms/uploads/attachment/file/538453/Comunicado_ Tecnico_Diario_COVID-19_2020.02.28.pdf

59. World Health Organization. Novel coronavirus situation report-2 [Internet]. 2020 [cited 2020 Apr 1]. Available in: www.who.int/ docs/default-source/coronaviruse/situation-reports/20200122sitrep-2-2019-ncov.pdf

60. Stadnytskyi V, Bax CE, Bax A, Anfinrud P. The airborne lifetime of small speech droplets and their potential importance in SARS-CoV-2 transmission. Proc Natl Acad Sci U S A. 2020; 117 (22): 11875-11877.

61. Ong SWX, Tan YK, Chia PY, Lee TH, Ng OT, Wong MSY et al. Air, surface environmental, and personal protective equipment contamination by severe acute respiratory syndrome coronavirus 2 (SARS-CoV-2) from a symptomatic patient. JAMA. 2020; 323 (16): 1610-1612.

62. van Doremalen N, Bushmaker T, Morris DH, Holbrook MG, Gamble A, Williamson BN et al. Aerosol and surface stability of SARS-CoV-2 as compared with SARS-CoV-1. N Engl J Med. 2020; NEJMc2004973.

63. Prevention $C$ for $D C$ and Clinical Guidance for Management of Patients with Confirmed 2019 Novel Coronavirus (2019-nCoV) Infection, Updated February 12, 2020 [Internet]. 2020 [cited 2020 Apr 1]. Available in: www.cdc.gov/coronavirus/2019ncov/hcp/clinical-guidance-management-patients.html

64. Chen W, Lan Y, Yuan X, Deng X, Li Y, Cai X et al. Detectable 2019-nCoV viral RNA in blood is a strong indicator for the further clinical severity. Emerg Microbes Infect. 2020; 9 (1): 469-473.

65. Tang A, Tong ZD, Wang HL, Dai YX, Li KF, Liu JN et al. Detection of novel coronavirus by RT-PCR in stool specimen from asymptomatic child, China. Emerg Infect Dis. 2020; 26 (6): 1337-1339.

66. Wang W, Xu Y, Gao R, Lu R, Han K, Wu G et al. Detection of SARS-CoV-2 in different types of clinical specimens. JAMA. 2020; 323 (18): 1843-1844.

67. WHO. Report of the WHO-China Joint Mission on Coronavirus Disease 2019 (COVID-2019). 2020.

68. Li Q, Guan X, Wu P, Wang X, Zhou L, Tong Y et al. Early transmission dynamics in Wuhan, China, of novel coronavirusinfected pneumonia. N Engl J Med. 2020; 382 (13): 1199-1207.

69. Chan JFW, Yuan S, Kok KH, To KKW, Chu H, Yang J et al. A familial cluster of pneumonia associated with the 2019 novel coronavirus indicating person-to-person transmission: a study of a family cluster. Lancet (London, England). 2020; 395 (10223): 514-523.

70. WHO. Coronavirus disease (COVID-19) situation report 128 data as received by WHO from national authorities by 10: 00 CEST, 27 May 2020 [Internet]. 2020 [cited 2020 May 27]. Available in: https://www.who.int/docs/default-source/ coronaviruse/situation-reports/20200527-covid-19-sitrep-128. pdf?sfvrsn=11720c0a_2

71. WHO. Coronavirus disease (COVID-19) outbreak situation, Last update: 27 May 2020, 19: 00 GMT-5 [Internet]. 2020 [cited 2020 May 4]. Available in: https://www.who.int/emergencies/ diseases/novel-coronavirus-2019

72. Team TNCPERE. The Epidemiological Characteristics of an Outbreak of 2019 Novel Coronavirus Diseases (COVID-19) - China, 2020. China CDC Weekly [Internet]. 2 (8): 113-22. Available in: http: //weekly.chinacdc.cn//article/id/e53946e2c6c4-41e9-9a9b-fea8db1a8f51

73. Guan WJ, Ni ZY, Hu Y, Liang WH, Ou CQ, He JX et al. Clinical characteristics of coronavirus disease 2019 in China. N Engl J Med. 2020; NEJMoa2002032. 
74. Wu C, Chen X, Cai Y, Xia J, Zhou X, Xu S et al. Risk factors associated with acute respiratory distress syndrome and death in patients with coronavirus disease 2019 pneumonia in Wuhan, China. JAMA Intern Med. 2020; e200994.

75. Holshue ML, DeBolt C, Lindquist S, Lofy KH, Wiesman J, Bruce $\mathrm{H}$ et al. First case of 2019 novel coronavirus in the United States. N Engl J Med. 2020; 382 (10): 929-936.

76. Bhatraju PK, Ghassemieh BJ, Nichols M et al. Covid-19 in critically ill patients in the Seattle region - case series. $\mathrm{N}$ Engl $\mathrm{J}$ Med. 2020; 382 (21): 2012-2022.

77. Richardson S, Hirsch JS, Narasimhan $M$ et al. Presenting characteristics, comorbidities, and outcomes among 5700 patients hospitalized with COVID-19 in the New York city area. JAMA. 2020; 323 (20): 2052-2059.

78. Secretaría de Salud. Información Internacional y Nacional sobre nuevo Coronavirus (COVID-2019) [Internet]. 2020 [citado 27 de mayo de 2020]. Disponible en: https://coronavirus.gob.mx/ datos/

79. Bello-Chavolla OY, Bahena-Lopez JP, Antonio-Villa NE, Vargas-Vázquez A, González-Díaz A, MárquezSalinas $A$ et al. Predicting mortality due to SARS-CoV-2: A mechanistic score relating obesity and diabetes to COVID-19 outcomes in Mexico. medRxiv [Internet]. 2020; 2020.04.20.20072223. Available in: http://medrxiv.org/content/ early/2020/05/05/2020.04.20.20072223.abstract

80. Mejia-Vilet JM, Cordova-Sanchez BM, Fernandez-Camargo D, Mendez-Perez RA, Morales-Buenrostro LE, Hernandez-Gilsoul T. Derivation of a score to predict admission to intensive care unit in patients with COVID-19: the ABC-goals score. medRxiv [Internet]. 2020; 2020.05.12.20099416. Available in: http: // medrxiv.org/content/early/2020/05/16/2020.05.12.20099416. abstract

81. Wang R, Liao C, He H et al. COVID-19 in hemodialysis patients: a report of 5 cases. Am J Kidney Dis. 2020. doi: 10.1053/j. ajkd.2020.03.009.

82. Ma Y, Diao B, Lv X, Zhu J, Liang W, Liu L et al. 2019 novel coronavirus disease in hemodialysis (HD) patients: report from one HD center in Wuhan, China. medRxiv [Internet]. 2020; 2020.02.24.20027201. Available in: http://medrxiv.org/content/ early/2020/02/27/2020.02.24.20027201.abstract

83. Xiong F, Tang H, Liu L, Tu C, Tian JB, Lei CT et al. Clinical characteristics of and medical interventions for COVID-19 in hemodialysis patients in Wuhan, China. J Am Soc Nephrol. 2020. doi: 10.1681/ASN.2020030354.

84. Wu J, Li J, Zhu G, Zhang Y, Bi Z, Yu Y et al. Clinical features of maintenance hemodialysis patients with 2019 novel coronavirus-infected pneumonia in Wuhan, China. Clin J Am Soc Nephrol. 2020; CJN.04160320. doi: 10.2215/ CJN.04160320.

85. Goicoechea M, Sánchez Cámara LA, Macías N, Muñoz de Morales A, González Rojas Á, Bascuñana A et al. COVID-19: Clinical course and outcomes of 36 maintenance hemodialysis patients from a single center in Spain. Kidney Int. 2020; S00852538(20)30509-3. doi: 10.1016/j.kint.2020.04.031.

86. Scarpioni R, Manini A, Valsania T, de Amicis $S$, Albertazzi V, Melfa L et al. Covid-19 and its impact on nephropathic patients: the experience at Ospedale "Guglielmo da Saliceto" in Piacenza. G Ital Nefrol. 2020; 37 (2).

87. La Milia V, Bacchini G, Bigi MC, Casartelli D, Cavalli A, Corti $M$ et al. COVID-19 outbreak in a large hemodialysis centre in Lombardy, Italy. Kidney Int Rep [Internet]. 2020. Available in: https://doi.org/10.1016/j.ekir.2020.05.019

88. Michaels MG, la Hoz RM, Danziger-Isakov L, Blumberg EA, Kumar D, Green M et al. Coronavirus disease 2019: implications of emerging infections for transplantation. Am J Transplant. 2020. doi: 10.1111/ajt.15832.

89. Kumar D, Tellier R, Draker R, Levy G, Humar A. Severe acute respiratory syndrome (SARS) in a liver transplant recipient and guidelines for donor SARS screening. Am J Transplant. 2003; 3 (8): 977-981.

90. AlGhamdi M, Mushtaq F, Awn N, Shalhoub S. MERS CoV infection in two renal transplant recipients: case report. Am J Transplant. 2015; 15 (4): 1101-1104.

91. Zhu L, Xu X, Ma K, Yang J, Guan H, Chen S et al. Successful recovery of COVID-19 pneumonia in a renal transplant recipient with long-term immunosuppression. Am J Transplant. 2020; ajt.15869.

92. Zhang $H$, Chen $Y$, Yuan $Q$, Xia $Q X$, Zeng XP, Peng JT et al. Identification of kidney transplant recipients with coronavirus disease 2019. Eur Urol. 2020; 77 (6): 742-747.

93. Zhu L, Gong N, Liu B, Lu X, Chen D, Chen S et al. Coronavirus Disease 2019 pneumonia in immunosuppressed renal transplant recipients: a summary of 10 confirmed cases in Wuhan, China. Eur Urol. 2020; 77 (6): 748-754.

94. Kim Y, Kwon O, Paek JH et al. Two distinct cases with COVID-19 in kidney transplant recipients. Am J Transplant. 2020. doi: 10.1111/ajt.15947.

95. Gandolfini I, Delsante M, Fiaccadori E, Zaza G, Manenti L, Degli Antoni A et al. COVID-19 in kidney transplant recipients. Am J Transplant. 2020.

96. Guillen E, Pineiro GJ, Revuelta I, Rodriguez D, Bodro M, Moreno A et al. Case report of COVID-19 in a kidney transplant recipient: Does immunosuppression alter the clinical presentation? Am J Transplant. 2020.

97. Fernández-Ruiz M, Andrés A, Loinaz C, Delgado JF, LópezMedrano F, San Juan R et al. COVID-19 in solid organ transplant recipients: A single-center case series from Spain. Am J Transplant. 2020.

98. Banerjee D, Popoola J, Shah S, Ster IC, Quan V, Phanish M. COVID-19 infection in kidney transplant recipients. Kidney Int. 2020; 97 (6): 1076-1082.

99. Alberici F, Delbarba E, Manenti C, Econimo L, Valerio F, Pola A et al. A single center observational study of the clinical characteristics and short-term outcome of 20 kidney transplant patients admitted for SARS-CoV2 pneumonia. Kidney Int. 2020.

100. Tschopp J, L'Huillier AG, Mombelli M, Mueller NJ, Khanna N, Garzoni $\mathrm{C}$ et al. First experience of SARS-CoV-2 infections in solid organ transplant recipients in the Swiss Transplant Cohort Study. Am J Transplant. 2020.

101. Abrishami A, Samavat S, Behnam B, Arab-Ahmadi M, Nafar M, Sanei Taheri M. Clinical course, imaging features, and outcomes of COVID-19 in kidney transplant recipients. Eur Urol. 2020

102. Akalin E, Azzi $Y$, Bartash $R$, Seethamraju $H$, Parides $M$, Hemmige $V$ et al. Covid-19 and Kidney Transplantation. N Engl J Med. 2020.

103. Nair V, Jandovitz N, Hirsch JS, Nair G, Abate M, Bhaskaran $M$ et al. COVID-19 in kidney transplant recipients. Am J Transplant. 2020.

104. Columbia University Kidney Transplant Program. Early Description of Coronavirus 2019 Disease in Kidney Transplant Recipients in New York. J Am Soc Nephrol. 2020; 31 (6): 11501156.

105. Pereira MR, Mohan S, Cohen DJ, Husain SA, Dube GK, Ratner LE et al. COVID-19 in solid organ transplant recipients: Initial report from the US epicenter. Am J Transplant. 2020. 
106. Machado DJB, lanhez LE. COVID-19 pneumonia in kidney transplant recipients-Where we are? Transpl Infect Dis. 2020; e13306.

107. Centro Nacional de Trasplantes (CENATRA). Boletín Informativo Especial Trasplantes y COVID-19. Información actualizada de los receptores de trasplantes y su condición respecto de la infección por SARS-CoV-2 [Internet]. 2020 [citado 27 de mayo de 2020]. Disponible en: https://www.gob.mx/cms/uploads/attachment/ file/553970/Bolet_n_Informativo_Especial_25-05-20.pdf

\section{Correspondencia:}

Dr. Ricardo Iván Velázquez-Silva Área de Nefrología del Trasplante.

Departamento de Nefrología y Metabolismo Mineral.

Instituto Nacional de Ciencias Médicas y Nutrición «Salvador Zubirán».

E-mail: ricardo.ivan.velaz@gmail.com 